\title{
The Dirac Equation in Low Energy Condensed Matter Physics
}

\section{John Swain*t}

Dept. of Physics, Northeastern University, Boston, MA 02115

E-mail: john. swain@cern.ch

\author{
Allan Widom \\ Dept. of Physics, Northeastern University, Boston, MA 02115 \\ E-mail: allan_widomeyahoo.com
}

\section{Yogendra Srivastava}

Department of Physics and Geology, University of Perugia, Perugia, Italy

Department of Physics, Northeastern University, Boston, MA 02115 USA

E-mail: yogendra.srivastava@gmail.com

Electrons in low energy condensed matter physics are typically treated using the non-relativistic Schrödinger or Pauli equations, with relativistic effects included, if at all, via corrections of order v/c. We show that using the full Dirac equation with 4-component spinors leads to a number of important qualitative effects and, perhaps surprisingly, some striking simplifications over the conventional lower energy treatments, already for nuclei with $\mathrm{Z}>2$.

38th International Conference on High Energy Physics

3-10 August 2016

Chicago, USA

* Speaker.

$\dagger$ J. S. thanks the National Science Foundation for support via NSF grant PHY-1205845. 


\section{Introduction}

The idea that the Schrödinger equation gives an adequate and indeed correct description of chemistry, from the periodic table to chemical bonding is rarely ever called into question. Dirac had famously quipped that the laws of physics as then understood could explain "most of physics and all of chemistry", with, at the time, chemistry being understood through the (non-relativistic) Schrödinger equation despite Dirac having discovered the correct (relativistic) equation which describes the electron. Richard Feynman, however, almost as famously quipped "There's a reason physicists are so successful with what they do, and that is they study the hydrogen atom and the helium ion and then they stop."

How well does the Schrödinger equation really describe the quantum mechanics of electrons in chemistry and, by extension, in condensed matter physics? In this paper we briefly review some history, starting with the original relativistic wave equation which Schrödinger had found, now called the Klein-Gordon equation, which was replaced by the non-relativistic Schrödinger equation, whose defects in matching experiment were then fixed, essentially by hand to mimic what one would get from a more correct treatment via the relativistic Dirac equation.

We then briefly review work done in "relativistic quantum chemistry" to incorporate corrections due to relativistic effects, noting the generally ignored but crucially important difference that comes about with relativity, which is that the labeling of states is radically different.

We then point out that, even when relativistic effects on energy levels are small, the change in angular distributions of electron orbitals due to the different labeling of states, and in particular, the fact that $\vec{L}$ and $\vec{S}$ are not separately conserved, is significant and has been worked around by ad-hoc means. Despite the additional complications involved in using a 4-component Dirac spinor in place of a single component Schrödinger wave function, certain important features of the angular distribution of electrons in atoms are almost obvious, and do not require the ad-hoc additions to the Schrödinger equation picture which normally must be made.

\section{The Quantum Mechanical Treatment of the Electron}

The Klein-Gordon equation, named for Oskar Klein and Walter Gordon in 1926 was proposed, along with many others, as possible wave equation for the electron. The equation apparently appears in Schrödinger's notebooks from late 1925, but it got the fine structure of the hydrogen atom wrong [1] and of course was plagued by negative energies. In 1926 Schrödinger submitted for publication[2] the nonrelativistic equation which bears his name, which reproduced the Bohr model energy levels, but without fine structure.

The orbitals, labelled by a radial quantum number $n$, orbital ones $\ell$ and $m$, and one representing spin $s$ are well-known even to high school students, and the entire structure of the periodic table is based on the extension of these "hydrogenic" wave functions. The energies depend only on $n$ are given by the well-known[3] expression

$$
E_{n}=-\frac{m_{e} c^{2} \alpha^{2}}{2 n^{2}}
$$

with $L^{2}=\vec{L} \cdot \vec{L}=\ell(\ell+1) \hbar^{2}, m$ running from $-\ell$ to $\ell$ in integer steps, and the spin $s= \pm \frac{1}{2} \hbar$. Here $m_{e}$ is the (reduced, to account for finite proton mass) electron mass, $c$ the speed of light, and $\alpha=\frac{e^{2}}{\hbar c}$ 
the fine structure constant. For atoms beyond hydrogen, with nuclei of charge $Z$, and only one electron, one replaces $\alpha$ with $Z \alpha$.

With more than one electron (and ignoring the complications of electron-electron interactions) a series of ad-hoc principles are applied[4]. First of all, a rule is invoked that only two electrons will be placed in a given wavefunction labelled by $n, \ell, m$. This is done via an appeal to the Pauli exclusion principle which cannot be derived from the Schrödinger equation, and indeed depends crucially on the electron having spin-1/2 (and, in fact, really requires a quantum field theoretic treatment[1]). This leads to a picture of "shells" which are filled in an order given by the $n+\ell$ rule, also known as the Madelung rule (after Erwin Madelung), or the Janet rule or the Klechkowsky rule (after Charles Janet or Vsevolod Klechkovsky, in some countries). Electrons in higher shells feel an effective screened nuclear charge $Z_{\text {eff }}$ due to electrons in lower shells, which is determined via Slater's rules $Z_{\text {eff }}=Z-\sigma$ for suitable $\sigma$. These $\sigma$ were determined semi-empirically by Slater[5] in 1930 and revised based on Hartree-Fock calculations by Clementi et al.[6] in the 1960's.

The essential point to note is that everything in chemistry and condensed matter is, in almost all cases, based on electron wavefunctions modeled on what is obtained by the non-relativistic Schrödinger equation for one electron and a charge $Z$ (or, rather, $Z_{\text {eff }}$ ) nucleus.

\section{The Dirac Equation}

The Dirac equation, which everyone would agree is actually the correct equation to describe electrons, is, unlike the Schrödinger equation, relativistic, which implies several major departures from the non-relativistic wavefunction description. First of all, it requires a 4-component wave function, the Dirac spinor, in order to describe electrons. The spin quantum number and its association with angular momentum of $\frac{1}{2} \hbar$ arises naturally, as does, via a quantum field theoretic treatment of the equation, the Pauli exclusion principle[1]. A key point to which we will return shortly is that the (non-relativistic) quantum numbers $\ell$ and $s$ no longer make sense, as neither the orbital angular momentum $\vec{L}$ nor the spin angular momentum $\vec{S}$ are separately conserved. That is, for the Dirac Hamiltonian $H_{D}$, neither $\left[H_{D}, \vec{L}\right]$ nor $\left[H_{D}, \vec{S}\right]$ vanish. However, the quantity $\vec{J}=\vec{L}+\vec{S}$ is conserved, and the value $j$ determined by $\vec{J} \cdot \vec{J}=j(j+1)$ is a good quantum number. Energy levels now depend not just on a radial quantum number $n$ but on $j$ as well, and

$$
E_{n j}=-m_{e} c^{2}\left[1-\left(1+\left[\frac{Z \alpha}{n-j-\frac{1}{2}+\sqrt{\left(j+\frac{1}{2}\right)^{2}-Z^{2} \alpha^{2}}}\right]^{2}\right)^{-\frac{1}{2}}\right]
$$

and it is not hard to see that for small $Z$ this gives much the same result as the Schrödinger equation, with no dependence on $j$.

While this expression is often well-approximated by the Schrödinger equation values, this is not always the case, and the corresponding radial wavefunctions can, for large enough $Z$, be affected significantly enough to have important quantitative and even qualitative effects. This leads to the subject of relativistic quantum chemistry which we touch on in the next section before coming to the central issue of this work which is the effect on the angular part of the wavefunction, which will turn out to be, though often ignored, very important to treat relativistically, even for low $Z$. 


\section{Relativistic Effects in Chemistry as Usually Treated}

The study of relativistic effects in chemistry is a well-advanced one (see, for example, the review by Pyykkö [7] or books such as[8, 9]). Relatively well-known examples of relativistic effects in chemistry are the nobility, trivalency[10] and yellow color of gold[11, 12], attributed to the contraction of the $6 \mathrm{~s}^{1}$ orbital. In fact, with the single outer $6 \mathrm{~s}$ electron, one might expect gold to behave more like an alkali metal rather than being the corrosion resistant metal we know it to be. The intuitive explanation often given for this is that the s-wave electron wave functions are more localized near the nucleus and thus feel a less screened charge than those in higher orbital angular momentum states and are more susceptible to relativistic effects.

McKelvey[13], writing for students, makes the simplistic and yet arguably charming argument that one can associate a speed $v=(Z \alpha) c$ by writing Bohr's quantization rule as $m v r=n \hbar$ and inserting the Bohr radius for $r$. Then for gold, with $Z=80$, a chemist can argue for a "relativistic mass" $m=m_{e} / \sqrt{1-\left(\frac{v}{c}\right)^{2}}$ of about $1.2 m_{e}$ which inserted into the Bohr formula implies a roughly $20 \%$ contraction of the 1s electron orbitals, as well as the other s orbitals since, citing Pyykko and Desclaux [11] "all the others shells, up to the valence shell, contract roughly as much because their electron speeds near the nucleus are comparable and the contraction of the inner part of the wave function pulls in the outer tails.". The low melting point of mercury[14] (also related to the fact that it is monatomic in the gas phase) is similarly explained by the contraction of the $6 \mathrm{~s}^{2}$ orbital so that it is mainly van der Waals forces between atoms. Rather remarkably, it has been recently shown[15] that for lead-acid batteries, over $1.7 \mathrm{~V}$ of the $2.1 \mathrm{~V}$ per cell comes from relativistic effects!

The rigorous approaches to relativistic quantum chemistry as are discussed in references such as $[7,8,9]$ all share one important feature: while they include relativistic corrections to the nonrelativistic Schrdinger equation in the form of additional spin and momentum dependent terms going to higher order in $v / c$, they invariably go to 2-component spinors and the usual (non-relativistic, we hasten to add) quantum numbers for orbital and spin angular momentum.

That this is a potentially dangerous thing to do is perhaps made most clear by the tendency to express the 4-component spinor in terms of "large" and "small" components, retaining the "large" ones and neglecting the "small" ones [3]. This gets one to the Schrödinger equation, now with a 2-component Pauli spinor which contains the spin information. However, the Dirac matrices can be chosen in any representation, and the distinction between "large" and "small" is, in fact, dependent on the representation. For example, in the chiral representation, there is no longer such a clear separation into "large" and "small". Even in the usual representation, the "small" components are no longer "small" as $Z$ becomes non-negligible compared to $1 / \alpha \approx 137$.

In fact, as is well-known [3], the correct (Dirac) hydrogenic wavefunctions, labelled by $j$, contain spherical harmonics $Y_{\ell m}$ with $\ell=j \pm \frac{1}{2}$, each of the two $\ell$ values appearing with a different spin. This simply indicates, as noted earlier, that $\ell$ and $s$ are not good quantum numbers and should not be used to label states. The fact that reasonable energy eigenvalues for the Hamiltonian can be achieved with wavefunctions with incorrect angular properties is perhaps not entirely surprising since the potential is spherically symmetric. The angular dependence of the wave functions, however, is not at all well-described by non-relativistic wavefunctions.

To the best of our knowledge, the simple fact that there are large qualitative differences in using the full relativistic treatment of angular momentum - quite independent of any relativistic 
effects on energy levels and the radial part of the wavefunction (such as those described above) has not been explicitly stated in the literature. It is to this we now turn.

\section{Relativistic Quantum Numbers and Angular Wavefunctions}

As noted above, the energy levels $E_{n j}$ for the relativistic (Dirac) electron depend on a radial quantum number $n$ and an angular one $j$. The correct quantum numbers [3] are $n, j$ and the parity $P$ of the state. For a high energy physicist this is immediately clear since one is accustomed to particles being labelled by, mass and so-called (colloquially) "spin-parity" or $J^{P}$.

We have already reviewed some of the ad-hoc additions needed to make the non-relativistic Schrödinger equation, which really describes spin- 0 particles, work for atomic physics. Now we describe another ad-hoc procedure, which is of enormous import, which also finds its honest origin in the use of the Dirac equation.

One of the most important atoms in the periodic table is undoubtedly carbon - a key building block for all life and perhaps the most important element in chemistry, with the entire subfield of organic chemistry devoted to it. This all arises from its ability to form 4 bonds with other atoms, exemplified in the tetrahedral structure of methane, or the carbon bonding in diamond.

Carbon has $Z=6$. The usual description of its orbital structure is $1 s^{2} 2 s^{2} 2 p^{2}$ meaning a filled $n=1, \ell=0$ s-shell with two electrons, spin up and spin down, and for $n=2$, a similarly filled spherical 2s orbital and 1 electron in each of 2 of the three roughly dumbbell-shaped p-orbitals, oriented along three perpendicular axes. However, in reality, this is not what is seen. Rather, one finds 4 orbitals with tetrahedral symmetry. This conundrum is resolved by a clever - but ad-hoc procedure, invented[16] by Linus Pauling in 1931, called hybridization. The idea is to form a linear combination of one $\mathrm{s}$ and three $\mathrm{p}$ orbitals to obtain agreement with experiment. This, however, is something which must be added by hand to the Schrödinger description to accommodate data.

Consider now the Dirac description. Despite the apparent complications of the Dirac equation, relativity, and 4-component spinors, one need only look at $j$ to understand this. The lowest possible value of $j$ is $1 / 2$ and corresponds to the non-relativistic $\ell=0$ state. There are $2 j+1$ or 2 electrons which can go in, one spin-up and one spin-down. The next value of $j$ is $3 / 2$ and we have $2 j+1=4$ states, all of the same energy. By symmetry they will be tetrahedrally oriented. Thought of nonrelativistically, they are made of "mixed" or "hybridized" $\ell$ values, but this in fact need not be done by hand. Rather, one now has a correct prediction instead of a problem with incorrect angular distributions that must be fixed by hand to get the right result.

This is a simple observation, but note that already for any $Z>2$ (where in the non-relativistic picture, $\ell>0$ ) we can expect significant discrepancies with the non-relativistic spin- 0 description, supporting quite clearly Feynman's comment about some of the apparent "success" he describes being indeed due to stopping at hydrogen and helium.

\section{Further Implications}

Now that we have made the simple observation that the Dirac equation should be used to describe the electron with orbital and spin angular momentum now replaced by $j$ and parity, this has many far-reaching implications. First of all, one should be careful with any work referring 
to "s-wave", "p-wave", "d-wave", etc. in chemistry or condensed matter physics which involves any angular dependence (even if correct angular distributions can be forced by ad-hoc means). The same sort of consideration applies to nuclear physics and in fact even to hadron spectroscopy, which notwithstanding the observations about $J^{P}$ made above, often calculate in terms of $L$ and $S$. An additional upside is that typically large " $\vec{L} \cdot \vec{S}$ " couplings now arise naturally whenever there is a $J^{2}$ involved. In a sense, part of the usual non-relativistic "Clebsching" of orbital and spin angular momenta is done automatically by the Dirac equation.

It is perhaps surprising that such an elementary observation with such important consequences has not been made before, but tradition and custom are powerful forces. An additional simple observation may help to make this all seem obvious (in hindsight): nobody would be surprised to find the angular dependence of scattering off a proton to be different for a spinless particle and a spin-1/2 particle. Given the relationship between poles in the scattering amplitude and bound states, it is then perhaps not surprising that angular distributions of bound-state wavefunctions would depend on the spin of what is being bound.

\section{References}

[1] C. Itzykson and J.-B. Zuber, “Quantum Field Theory”, McGraw-Hill Co. (1985).

[2] E. Schrödinger, Ann. Physik (4) 79361 (1925); 79489 (1925); 80437 (1926); 81109 (1926).

[3] A. Messiah, "Quantum Mechanics", Dunod, Paris (1995).

[4] See, for example, Gary L. Miessler, Donald A. Tarr, "Inorganic Chemistry", Prentice Hall (2003).

[5] J. C. Slater, Phys. Rev. 36 (1) 57 (1930).

[6] Clementi, E.; Raimondi, D. L. (1963) J. Chem. Phys. 38 (11) 2686 (1963); Clementi, E.; Raimondi, D. L.; Reinhardt, W. P. (1967) Journal of Chemical Physics 47 (4): 1300 (1967).

[7] P. Pyykkö, Chemical Reviews, 112371 (2012).

[8] K. G. Dyall and K. Fægri, Jr., "Relativistic Quantum Chemistry”, Oxford University Press, New York (2007).

[9] K. Balasubramanian, "Relativistic Effects in Chemistry Part A", John Wiley \& Sons, New York (1997); "Relativistic Effects in Chemistry Part B", John Wiley \& Sons, New York (1997).

[10] P. J. Schwerdtfeger, Am.Chem.Soc. 1117261 (1989).

[11] P. Pyykkö and J. P. Desclaux, Acc. Chem. Res. 12276 (1979)

[12] P. Romaniello and P. L. de Boeij, J. Chem. Phys. 164303122 (2005); J. Chem. Phys. 17411127 (2007).

[13] D. R. McKelvey, J. Chem. Educ. 60112 (1982).

[14] P. Pyykkö, Adv. Quantum Chem. 11353 (1978).

[15] R. Ahuja et al., Phys. Rev. Lett. 106018301 (2011).

[16] L. Pauling, Journal of the American Chemical Society 53 (4): 1367 (1931). 